\title{
Novel insights on biomineralization using x-rays and optical coherent diffraction based imaging approaches
}

\author{
V. Chamard \\ Aix-Marseille Univ, CNRS, Centrale Marseille, Institut Fresnel, Marseille, France; \\ virginie.chamard@fresnel.fr
}

Biomineralization is the fascinating capability of living organisms to produce hard tissue. It integrates complex physical and chemical processes controlled by the organisms through ionic concentration regulation and organic molecules production. The ability to tune, from ambient conditions crystallisation, the structural, optical and mechanical properties of these hard tissues motivates extensive research to develop and transfer biomimetic approaches into material science studies. In paleoclimatology, where marine biomineral tests are used as paleo-tracers, understanding the biomineralization mechanisms is a key factor for improving the accuracy of thermal records. All these urge a detailed description of the underlying processes at play in biomineralisation.

Remarkably, most crystalline biominerals presents a sub-micrometric organo-mineral granular organization associated to crystallization kinetics, which involve different mineral polymorphs. While these features clearly point towards biomineralisation mechanisms escaping from the classical crystallisation theory (i.e., described as the addition of individual ions or molecules from a solution to a final bulk crystal), the specific pathways are however still subject to intense debates [1]. Providing a full description of the biomineralization pathways requires a multidisciplinary approach at the interface between biology, chemistry and physics, in order to identify not only the different minerals and organic molecules involved along the mineralization process, but also characterize the morphology of these constituents, the successively appearing polymorph phases and the nature of the different phase transitions. In this presentation, I will focus on this last aspect, making use of two crystalline microscopy approaches we have developed, optical vectorial ptychography [2] and x-ray Bragg ptychography [3].

Optical vectorial ptychography is a microscopy approach sensitive to the optical anisotropy of materials, among which birefringence. In the context of biomineralisation, it was used to characterize extended $2 \mathrm{D}$ crystals and evaluate their crystalline properties (orientation distribution, disorder, etc...), thanks to a lab-based experimental set-up. X-ray Bragg ptychography requires the use of a nano-diffraction set-up at a synchrotron source (such as ID13 - ESRF) and delivers 3D maps of crystalline properties, including strain, tilts and crystalline coherence. With these crystalline microscopy approaches, we were able to shed new light on the characteristics of early-mineralized units from calcareous mineralizing specie, the Pinctada margarita mollusc shell. The confrontation of the observed crystalline properties with the ones obtained on well-defined synthetic model films allowed us to discuss the nature of the amorphous to crystalline transition in biominerals.

[1] J. J. De Yoreo, et al., Science 349, aaa6760 (2015).

[2] P. Ferrand, et al., Optics Letters 43, 763 (2018).

[3] F. Mastropietro, et al., Nature Materials 16, 946 (2017). S. O. Hruszkewycz, et al., Nature Materials 16, 244 (2017).

\section{Keywords: Biomineralisation; Crystallisation; Bragg ptychography; Vectorial ptychography; Calcareous species}

This project has received funding from the European Research Council (ERC) under the European Union's Horizon H2020 research and innovation program grant agreement No 724881. The author thanks all collaborators from Fresnel Institute, CEA-NIMBE, Ifremer and ESRF for their participation to this work. 